\title{
Personality, (NEURO) Trust and LMX in Information Apportionment
}

\author{
Chhaya Wadhwa, Ruchi Malik, J. Satpathy
}

\begin{abstract}
Neuromanagement has evolved into a full-fledged enterprise of consilience; an attempt to not only test and bridge, but truly unify natural science and social science explanations of human behaviour (Wilson, 1998; Glimcher and Rustichini, 2004; Rangel, Camerer and Montague, 2008). This paper presents an empirical approach to neuro - based information sharing dynamics via personality, (neuro) trust and LMX. The Big Five Inventory matrix has been adopted to test the phenomenon. A neuro - based pathway has been projected. A symbiotic relational model has been arrived at.
\end{abstract}

Keywords: Data / Neuro - Based Information / Fact Sharing, Personality Traits, (Neuro) Trust and LMX.

\section{INTRODUCTION}

$\mathrm{I}_{\mathrm{t}}$ is an undebated proposition that all neural activities are causally underlies humanoid cognition and behaviour. In the present world of business and management of business (es), 'data-driven' decision making backdrop has assumed the unparalled dimension and catalytic role of steering organisations to change contemporary and prevailing progressions thereby forcing businesses to change their fundamental foundations. Organisations nowadays are under unswerving forces of VUCA (vulnerability, uncertainty, complexity and ambiguity), coupled up with risks, probability and possible prospects that (may) even take form of mergers, acquisitions, restructuring etc. Henceforward, in present day dynamic environment, data / neuro - based information / fact sharing is extremely consequential element for any organisation (Cao and Xiang, 2012). In today's globally competitive environment, organisations are striving hard to create a culture of data / neuro - based information / fact sharing via various data / neuro - based information / fact management databases. These databases are fetching part and parcel of training modules in organisations. However, efficacy of these data / neuro - based information / fact management databases can be questionable as there are several factors that contribute towards data / neuro - based information / fact sharing. Thus, it becomes imperative to study antecedents of data / neuro - based information / fact sharing (Cabrera and Cabrera, 2002).

Revised Manuscript Received on December 05, 2019.

* Correspondence Author

Prof. (Dr.) Chhaya Wadhwa*, Assistant Professor, Apeejay School of Management, New Delhi, India.

Ruchi Malik, Assistant Professor, Apeejay School of Management, New Delhi, India.

Col (Dr.) J. Satpathy, Faculty, National Defence Academy, Pune, India.
Sonum bonum, this directly enhances organisational output and efficacy (Peralta and Saldanha, 2014, Nonaka, 1994; Nonaka and Takeuchi, 1995; Quigley, Tesluk, Locke, and Bartol, 2007). Literature suggests that data / neuro based information / fact sharing, within any organisation, makes the employees more adaptive to newer and newer changes and hence, resulting in much better value creation (Mafabi, Munene, and Ntayi, 2012). Approximately, antecedents of data / neuro - based information / fact sharing can be divided into two factors: individual and organisation factors.

\section{AIM AND SCOPE}

Cao and Xiang (2012) suggested that in order to have more efficient data / neuro - based information / fact sharing process in the organisation, leaders should evolve with a creative incentive database. For the present study, individual factors that influence data / neuro - based information / fact sharing are empirically examined though personality traits. Some organisational factors are empirically studied through (neuro) trust and LMX (Leader member Exchange) model. However, this alone does not suffice efficient data / neuro based information / fact sharing processes within an organisation. Reason is that it involves individuals who may have different personality traits (Fang et al., 2013). Individual's own readiness to share data / neuro - based information / fact depends on his or her personality traits and also, (neuro) trusts in the organisation and organisation's leader approach towards data / neuro - based information / fact sharing. Therefore, it becomes imperative to study these three main yardsticks of data / neuro - based information / fact management process, viz. personality traits, (neuro) trust and leader member exchange LMX.

\section{A. Personality Traits}

It is reliably agreed that the personality dimension of personality traits has been hypothesised to be predisposed by levels of dopamine in central nervous system. Personal psychology has engrafted itself as a pillar of Individual's personality. It plays a vital role towards data / neuro - based information / fact sharing intention in the organisation (Wadhwa and Bali, 2016). The theories of personality traits are beginning to set in a set of well - defined innovative questions to existing dynamics via the inter - disciplinary mode. The classical Big Five Model, encompassing perceptions into constancy, heritability, relational discernment, and penalties of disposition characteristics, among other things, defines five core aspects of personality which include extraversion, neuroticism, and openness to experience, agreeableness, and conscientiousness (Cost McCrae 1992; Duff, Boyle,

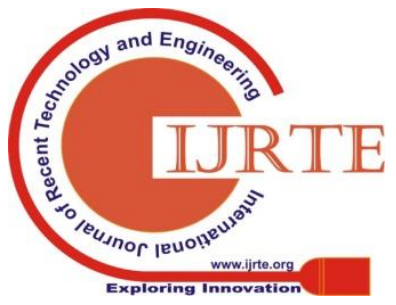


Dunleavy, Ferguson, 2004; Petrides, et al 2010).

For the present study only two parameters are considered: agreeableness and conscientiousness. Agreeableness measures the degree to which people are supportive, (neuro) trusting, helpful, conflict-avoiding, and enjoy the company of others. If managers are low on agreeableness, data / neuro - based information / fact sharing among them would yield negative results. On the other hand, people with high agreeability will exhibit openness, simplicity, sophistication and conviction (Costa and McCrae, 1992). Borges (2013) defined conscientiousness as the degree, to which an individual tends to be responsible, dependable, persistent, and work-oriented (Latham and Pinder, 2005). Conscientiousness is a personality trait that connotes being self disciplined, dutiful and competent (McCrae and Costa, 1986). Individuals who have a high score on conscientiousness are likely to be task oriented, self motivated, achievement oriented and an initiator (Witt et al., 2002) and consequently, they are expected to be more willing to share data / neuro - based information / fact (Matzler et al., 2011).

\section{B. (Neuro) Trust}

As extension of psychophysics and perception, (neuro) trust encompasses sensory procedures, awareness, consideration and psychophysics, methods, techniques and instrumentation. As extension of behaviour, (neuro) trust encompasses emotion, cognition, and other psychological systems, with an emphasis on behavioural perspectives. (Neuro) trust is the firm belief in the reliability or in the ability of someone or something. Literature suggests that (neuro) trust in supervisor is the basic foundation towards building a positive perception of the supervisorial characters and competencies in the minds of employees (Byrne et al., 2012). (neuro) trust is an employee's satisfaction in organisational decisions regarding expectation of fairness for the contribution to the firm (Reychav and Sharkie, 2010). The supportive leader's behaviours create a (neuro) trusting and safe environment promotes prosocial behaviours. (Neuro) trust is a vital parameter in an organisation's intent towards data / neuro - based information / fact sharing. This is because (neuro) trust enhances confidence, reduces the feeling of vulnerability and mitigates fear among employees in the organisation. If there is mutual (neuro) trust in the organisation, the intention towards data / neuro - based information / fact sharing blossoms manifolds (Dzandu et al., 2014).

\section{Leader-Member Exchange (LMX)}

LMX is a two-way, dyadic relationship between the leader and the subordinate. The LMX theory asserts that the supervisors do not treat their subordinates uniformly. (Graen and Cashman, 1975; Wayne and Green, 1993). The leader exchanges his personal and positional power of decision-making, task assignment, job latitude, support, and attention, differently at different degrees and levels due to the leader's limited time and resources, in return for subordinates' performance. (Graen and Cashman, 1975).
There is a high quality relationship with the 'in-group' subordinates who are willing to go out of their way to perform their tasks in accordance with the employment contracts have earned the (neuro) trust of the supervisors and can be counted upon by them to perform unstructured work and go the extra mile for them. Graen and Uhl-Bien, 1995, in their research posit that as a consequence of this differential treatment, the members of the in-group, with high quality LMX relationships are likely to receive more benefits from their supervisors (Wang et al., 2005). Consequently they display mutual positive support, informal interdependencies, and shared loyalty and thus may choose to share data / neuro based information / fact as a means of reciprocity towards their supervisors based on social exchange theory.

Therefore, it is predicted that agreeableness and conscientiousness traits of personality, (neuro) trust and LMX would determine the data / neuro - based information / fact sharing intention of the employees. Data / neuro based information / fact sharing intent is a function of Personality traits, (neuro) trust and LMX . Data / neuro based information / fact sharing intention (dependent variable) can be explained by Personality, (neuro) trust and LMX (independent variable). The general linear model equation is:

$$
\mathrm{Y}=\alpha+\beta 1 X 1+\beta 2 \mathrm{X} 2+\beta 3 \mathrm{X} 3+\varepsilon \mathbf{i}(1)
$$

There are three predictor terms $(\beta 1$ through $\beta 3)$ : Personality traits (X1), (neuro) trust (X2), and the Leader Member Exchange LMX (X3).

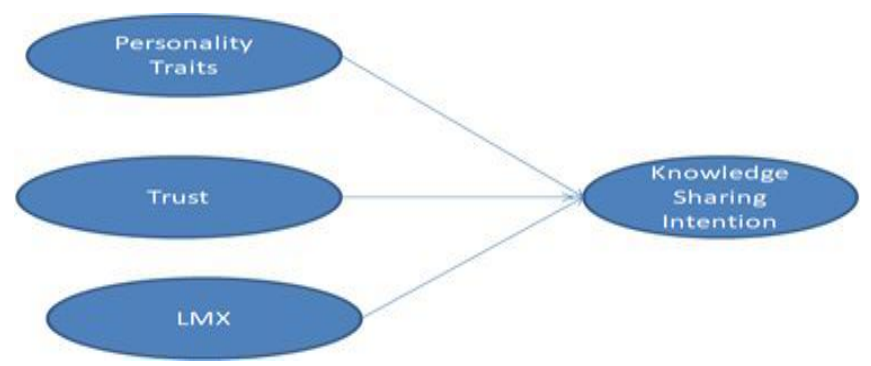

Figure - 1: General Linear Analysis Model

\section{METHODOLOGY}

\section{A. Measures}

Quantitative measures were used for the data collection. These were used to measure the big five personality variables, Leader member exchange, (neuro) trust and attitude of employees towards data / neuro - based information / fact sharing. Demographic variables related to age, gender, occupation, tenure in the organisation, etc were asked from the participants. For each construct, the total score was obtained for each participant by aggregating the item scores for each measure except for the demographic details. Established scales with high reliability were used to measure the study variables. Five point (1-5) Likert scales with anchors ranging from strongly disagree to strongly agree were used for all the items. Two dimensions of the big five personality - agreeableness and conscientiousness, were taken into consideration, and were measured using the Big

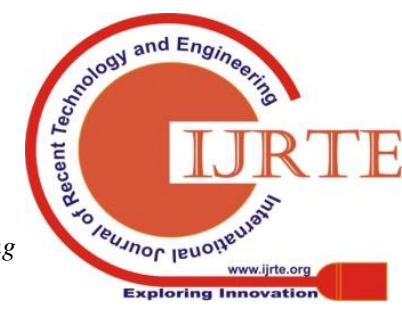


Five Inventory (Benet-Martinez and John, 1998), the Cronbach alpha was 0.79 . Items measuring the attitude towards Data / neuro - based information / fact sharing were adapted from Cheng and Chen (2007), and the Cronbach of the scale was 0.81 .

(neuro) trust was measured using the Employee (neuro) trust scale (Kim and Lee, 2010), with Cronbach alpha of the scale being 0.84, Leader-member exchange (LMX) was measured using a seven-item scale developed by Scandura and Graen (1984) and the Cronbach alpha of the scale was 0.78 .

\section{B. Sample and Procedures}

Value of data / neuro - based information / fact sharing in understood and exhibited when employees are encouraged and incentivized to express, share, negotiate, and understand tacit data / neuro - based information / fact. Managers working at the mid level and senior level working in various organisations in the Delhi NCR were asked to fill the survey and 307 responses were finally used for the analysis. The participants were advised that participation in this study was completely voluntary. They were also told that the strict confidentiality of the data would be maintained and data from this research would be reported only in the aggregate. For testing the model linear multiple regressions using SPSS 22 was used.

Table - 1: Demographic Profile of Respondents

\begin{tabular}{|c|c|c|}
\hline \multicolumn{1}{|c|}{ Profile } & $\begin{array}{c}\text { Frequency } \\
\mathbf{N = 3 0 7}\end{array}$ & Percentage \\
\hline Gender & & \\
\hline Male & 167 & 54.39 \\
\hline Female & 140 & 45.60 \\
\hline Age (In Years) & & \\
\hline Less Than 30 Years & 97 & 31.59 \\
\hline 31-40 Years & 112 & 36.48 \\
\hline $41-50$ Years & 56 & 18.24 \\
\hline $51-60$ Years & 42 & 13.68 \\
\hline
\end{tabular}

\section{RESULTS AND ANALYSIS}

In order to check the robustness of the proposed model that data / neuro - based information / fact sharing intention is a function of Personality traits (agreeableness and conscientiousness), (neuro) trust and Leader Member Exchange LMX, linear multiple regression analysis was conducted. On conducting a regression of the dependent variable the attitude towards data / neuro - based information / fact sharing of the participants and the independent variables, it was revealed that these variables are able to explain $49 \%$ variance $(r$ square change $=.492$ ) in the attitude towards data / neuro - based information / fact sharing.

Table - 2: Model Summary of Results

\begin{tabular}{|c|c|c|c|c|} 
Model & $\mathrm{R}$ & $\begin{array}{c}\mathrm{R} \\
\text { Square }\end{array}$ & $\begin{array}{c}\text { Adjusted } \mathrm{R} \\
\text { Square }\end{array}$ & $\begin{array}{c}\text { Std. error of the } \\
\text { Estimates }\end{array}$ \\
\hline 1 & $0.701 \mathrm{a}$ & 0.492 & 0.487 & 1.03 \\
\hline
\end{tabular}

a. Predictors: (Constant), Agreeableness, conscientiousness, LMX, (neuro) trust

\section{CONCLUSION}

Data / neuro - based information / fact management is a multi disciplinary approach having symbiotic and synergistic impact on organisation by efficient data and neuro - based information creation and neuro - based information sharing. From the study it can be deducted that data / neuro - based information / fact management behaviour is a key to success for any growing organisation. There are several variables that contributed in the efficacy of data / neuro - based information / fact management behaviour. However, for the present study three variables are considered which is able to explain almost fifty percent of the model. This clearly depicts that a huge percentage of causality get explained by personality traits, (neuro) trust and leader-member exchange. The organisations need to create a culture of data / neuro - based information / fact sharing by taking appropriate measures. These may include: firstly, looking at the individual personality traits prior to recruiting him and secondly by creating an environment of mutual (neuro) trust and respect within the organisation. This aspect does not happen overnight but requires unswerving effort on part of the employer as well as the supervisor. Lastly, organisations should also develop a more positive environment of leader member neuro - based information exchange. Technology can further facilitate this task of data / neuro - based information / fact sharing behaviour. Hence, data / neuro based information / fact sharing is a major catalyst towards organisation's future and success.

\section{RECOMMENDATIONS}

This research focuses on the relationship of personality traits, (neuro) trust and LMX on data / neuro - based information / fact sharing intention. This research can further be extended in several ways and the results may further enhance the organisational performance. Firstly, this study focuses on examining the relationship and the causality of (neuro) trust, personality, and LMX only on data / neuro based information / fact-sharing behaviour. There are several other variables that can be considered to have a better model fit. Specially, technology and automation as an antecedent can be incorporated as one of the causal factors. Secondly, this research can further be extended to different sectors in the economy to see the significant relationships across different variables. 


\section{Personality, (NEURO) Trust and LMX in Information Apportionment}

\section{REFERENCES}

1. Bock, G. W., Zmud, R. W., Kim, Y. G., and Lee, J. N. (2005). Behavioural intention formation in data based information / fact sharing: Examining the roles of extrinsic motivators, social-psychological factors, and organisational climate. MIS quarterly, 29(1), 87-111.

2. Cao, Y., and Xiang, Y. (2012). The impact of data based information / fact governance on data based information / fact sharing. Management Decision, 50(4), 591-610.

3. Dysvik, A., Buch, R., and Kuvaas, B. (2015). Data based information / fact donating and data based information / fact collecting: The moderating roles of social and economic LMX. Leadership and Organisation Development Journal, 36(1), 35-53.

4. Fang, Y., Wade, M., Delios, A., and Beamish, P. W. (2013). An exploration of multinational enterprise data based information / fact resources and foreign subsidiary performance. Journal of World Business, 48(1), 30-38.

5. Ferreira Peralta, C., and Francisca Saldanha, M. (2014). Data based information / fact-centered culture and data based information / fact sharing: the moderator role of () trust propensity. Journal of Data based information / fact Management, 18(3), 538-550.

6. Kim, S. L., Han, S., Son, S. Y., and Yun, S. (2017). Exchange ideology in supervisor-subordinate dyads, LMX, and data based information / fact sharing: A social exchange perspective. Asia Pacific Journal of Management, 34(1), 147-172.

7. Mafabi, S., Munene, J., and Ntayi, J. (2012). Data based information / fact management and organisational resilience: Organisational innovation as a mediator in Uganda parastatals. Journal of Strategy and Management, 5(1), 57-80.

8. Mooradian, T., Renzl, B., and Matzler, K. (2006). Who () trusts? Personality, () trust and data based information / fact sharing. Management learning, 37(4), 523-540.

9. Nonaka, I. (1994). A dynamic theory of organisational data based information / fact creation. Organisation science, 5(1), 14-37.

10. Nonaka, I., and Takeuchi, H. (1995). The data based information fact-creating company: How Japanese companies create the dynamics of innovation. Oxford university press.

11. Okyere-Kwakye, E., and Nor, K. M. (2011). Individual factors and data based information / fact sharing. American Journal of Management and Business Administration, 3(1), 66-72.

12. Quigley, N. R., Tesluk, P. E., Locke, E. A., and Bartol, K. M. (2007). A multilevel investigation of the motivational mechanisms underlying data based information / fact sharing and performance. Organisation science, 18(1), 71-88.

13. Staples, D. S., and Webster, J. (2008). Exploring the effects of () trust, task interdependence and virtualness on data based information / fact sharing in teams. - based information Systems Journal, 18(6), 617-640.

14. Tohidinia, Z., and Mosakhani, M. (2010). Data based information / fact sharing behaviour and its predictors. Industrial Management and Data Systems, 110(4), 611-631.

15. Wadhwa, C., and Bali, M. (2016). Antecedent of data based information / fact sharing intentions: An exploratory study. FIIB Business Review, 5(4), 65-73.

16. Zhou, K. Z., and Li, C. B. (2012). How data based information / fact affects radical innovation: Data based information / fact base, market data based information / fact acquisition, and internal data based information / fact sharing. Strategic management journal, 33(9), 1090-1102.

\section{AUTHORS PROFILE}

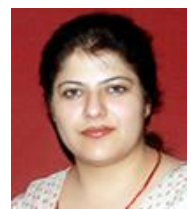

Dr. Chhaya Wadhwa, PhD, MBA, MPhil, MA, UGC NET, is an Assistant Professor (Communications \& Human Resource ) at ASM . Her Area of Interest is Recruitment Communication, Work Life Balance, Knowledge Management, etc. She has published several research papers in national and international journals of repute.

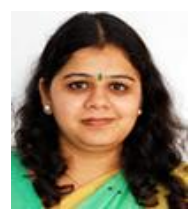

Ms. Ruchi Malik, is an Assistant Professor (Marketing) at ASM. Her area of interest is Consumer Behavior, Marketing Research, Relationship Marketing and Retail Management. She is an M.Phil., MBA , UGC-NET (Management) \& B.A .Economics (H). She has published several research papers in national and international journals of repute.

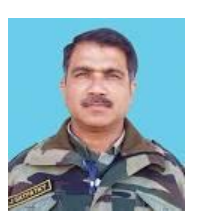

Colonel (Dr.) J. Satpathy, Academics Department, National Defence Academy, Pune . He is Double MPhil, Triple PhD, Quadruple D'Litts, AMT / AIMA, Faculty and Post - Doc in Neuromanagement. ). He has published several research papers in national and international journals of repute. 